\title{
Gamma-Ray Blazars and Active Galactic Nuclei seen by the Fermi-LAT
}

\section{Benoît Lott* on behalf of the Fermi/LAT Collaboration}

Centre d Etudes Nucléaires de Bordeaux Gradignan, CNRS/IN2P3, Université de Bordeaux, UMR 5797, Gradignan, 33175, France

E-mail: lottecenbg.in2p3.fr

The Second Catalog of Blazars and other AGNs detected by the Fermi/LAT (2LAC) includes about 1100 sources, 886 of which comprise the Clean Sample. The general properties of the different populations of sources classified according to the strength of their emission lines (FSRQs, BL Lacs) or the estimated position of the synchrotron peak are reviewed. Features of these sources in the radio range are discussed.

11th European VLBI Network Symposium \& Users Meeting 9-12 October 2012

Bordeaux (France)

\footnotetext{
* Speaker.
} 


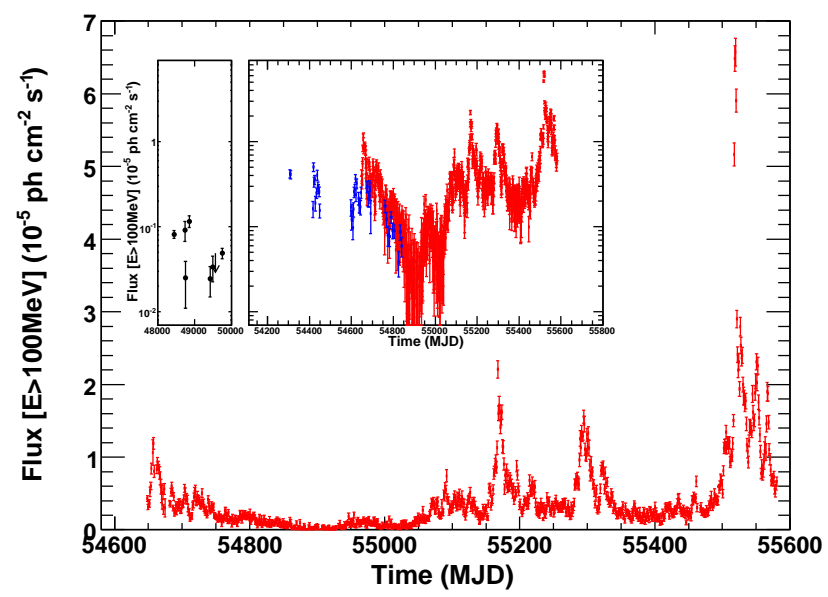

Figure 1: Daily light curve of 3C454.3 measured with the Fermi-LAT since launch. Inset: Historical light curve. Black points are from EGRET [2] and blue points are from AGILE [3].

\section{Introduction}

Since the launch of the Fermi satellite in June 2008, the Fermi-LAT has opened a new era in high-energy astrophysics. The LAT instrument, built by an international consortium of institutions from the USA, Italy, France, Sweden and Japan, is composed of 16 elements each including a tracker and a calorimeter. The whole instrument is surrounded by an anticoincidence detector, allowing a discrimination between gamma-ray photons and background charged cosmic rays. The LAT energy range is $20 \mathrm{MeV}-300 \mathrm{GeV}$, the high-energy end corresponding to a statistical shortage of detected photons. The point-spread function of the detector is strongly energy dependent. The field of view is 2.4 sr, i.e., about $20 \%$ of the entire sky is covered at a given time. The orbit altitude is $565 \mathrm{~km}$, corresponding to a period of about 91 minutes. The orbit inclination is $25.6^{\circ}$. In survey mode, the instrument rocks by about $50^{\circ}$ altenatively south and north over one orbit, enabling the whole sky to be scanned every 3 hours with roughly uniform exposure.

\section{The variable sky seen by the LAT}

The LAT presents many assets regarding AGN Science. Its unprecedented sensitivity, almost uniform at high galactic latitude, makes it very suitable to study populations of extragalactic objects. The continuous scan in survey mode allows alerts to be issued shortly after transient or new flaring sources are detected and enables as well source monitoring on time scales ranging from months down to a few hours. One of the most spectacular examples concerns the flares of the blazar 3C 454.3 (Fig. 1), which reached a record flux level above $100 \mathrm{MeV}$ of $8 \times 10^{-5} \mathrm{ph} \mathrm{cm}^{-2}$ $\mathrm{s}^{-1},\left(\gamma\right.$-ray luminosity $\left.>10^{50} \mathrm{erg} / \mathrm{s}\right)$ showing variability time scales of a few hours [1].

Another breakthrough arises from the Fermi/LAT large energy range extending into the 10-100 $\mathrm{GeV}$ domain, which was previously little explored. This feature has allowed new spectral features at high energy to be discovered and has also enabled the identification of potential candidates of $\mathrm{TeV}$ sources, effectively leading to several discoveries by ground-based Cherenkov detectors. 
At the time of this conference, about 130 Astronomers Telegrams (ATels) have been issued on the basis of LAT data, of which 120 concerned AGNs. The alert threshold is a flux above $100 \mathrm{MeV}$ greater than $10^{-6} \mathrm{ph} \mathrm{cm}^{-2} \mathrm{~s}^{-1}$, but other considerations like a significant flux rise with respect to the average flux, the detectability at $\mathrm{TeV}$ energies for a known source or the rarity of a transient event come into play when issuing an Atel. The "Flare Advocates" constantly monitor the sky, issue alerts and feed the Fermi blog. The list of ATels is posted at http://www-glast.stanford.edu/cgibin/pub_rapid.

As EGRET demonstrated that the extragalactic $\gamma$-ray sky was dominated by blazars, there were great expectations concerning advances in blazar and AGN science from the Fermi/LAT. Many of these expectations have been or are about to be fulfilled. In the following, recent advances concerning Fermi/LAT blazar populations and their different properties are presented.

\section{Blazar Populations}

The Second Catalog of Active Galactic Nuclei detected by the Fermi/LAT [4], referred to as 2LAC in the following is a spinoff of the Fermi/LAT Second Source Catalog (2FGL) [5] based on data accumulated over the first 24 months of sky survey. A first list of Fermi/LAT-detected AGNs using 3 months of data and including 106 high-confidence sources, called the LAT Bright AGN Sources (LBAS) [6] was released in early 2009. The First LAT AGN Catalog (1LAC) [7] was released in the Fall of 2010. It made use of 11 months of data and included 709 sources.

The mean $95 \%$ containment radius is $0.15^{\circ}$ for the high-latitude 2FGL sources. The association of a given source with an AGN is based on spatial coincidence following three different approaches described in [4]. Different catalogs were used for this purpose including the Combined Radio All-sky Targeted Eight GHz Survey (CRATES) [8], the Candidate Gamma-Ray Blazar Survey (CGraBs) [9] and the Roma-BZCat [10]. This procedure leads to the association of 1017 sources at $|b|>10^{\circ}$ (with association probability determined from Monte-Carlo simulations $P_{i}>0.8$ ) corresponding to 991 distinct $\gamma$-ray sources (out of 1319 2FGL sources lying in this galactic-latitude range). A total of 886 sources belong to the "Clean Sample" meeting the following conditions: no double associations and no anomaly observed in the association or detection procedures. Only roughly 12 associations are expected to be spurious in that sample. The validity of the procedure is confirmed by the good agreement of the actual distribution of angular separation between $\gamma$-ray sources and their AGN counterparts with theoretical expectations.

Although the fraction of identification of high-galactic sources is fairly large $(\simeq 75 \%)$, some identifications are clearly missed as a significant asymmetry between northern and southern galactic hemispheres is observed (only $38 \%$ of BL Lacs are observed at $b<-10^{\circ}$, while little asymmetry is observed for the whole 2FGL sources), manifesting the incompleteness of the parent catalogs.

The classification in terms of Flat-Spectrum Radio Quasars (FSRQs) and BL Lac-type objects (BL Lacs) is conventionally based on the strength of the emission lines. This classification has been used in both the 1LAC and the 2LAC. In addition, a more refined classification based on the source spectral energy distribution (SED) and dealing with the location of the synchrotron peak $v_{s y n}$ has been carried out by using archival data at lower wavelengths, more precisely $\alpha_{r o}$ and $\alpha_{o x}$, the radioto-optical and optical-to-X-ray spectral slopes. The empirical relation between $\left(\alpha_{r o}, \alpha_{o x}\right)$ and $v_{s y n}$ was derived from a set of 48 high-quality, simultaneous SEDs of $\gamma$-ray blazars [12]. Sources were 


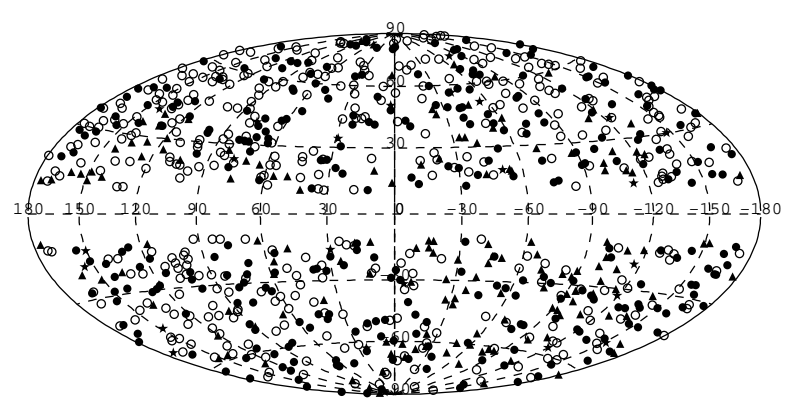

Figure 2: Locations of sources in the 2LAC Clean Sample. Solid circles: FSRQs, open circles: BL Lacs, stars: radio galaxies, triangles: AGNs of unknown type.

classified as low-, intermediate- or high-synchrotron peaked sources (referred to as LSPs, ISPs, HSPs respectively $)$ if $\log \left(v_{\text {syn }} / H z\right)<14,14<\log \left(v_{\text {syn }} / H z\right)<15$ or $\log \left(v_{\text {syn }} / H z\right)>15$ respectively.

The census of sources in the 2LAC Clean Sample is as follows: 310 FSRQs, 395 BL Lacs (44\% of which have a measured redshift), 24 other AGNs, 157 of unknown type. The more frequent detections of BL Lacs relative to FSRQs is in sharp contrast with the situation encountered with EGRET [2] where FSRQs outnumbered BL Lacs by a factor $\simeq 3$. This is probably primarily related to the larger sensitivity at high energy of the LAT, as most BL Lacs have harder spectra than FSRQs as discussed below. Moreover, the LAT is far more sensitive to multi-GeV photons than was EGRET, which lost sensitivity above several GeV due to self-vetoing effects. Since the 2LAC was realeased, Shaw et al. [11] have obtained redshift constraints on most BL Lacs missing redhifts. Only $5 \%$ of the 2 LAC BL Lacs remain unconstrained.

The 24 non-blazar objects in the 2LAC Clean Sample include 8 misaligned AGNs, 4 NarrowLine Seyfert 1 galaxies, 10 AGNs of other types and 2 starburst galaxies. A total of 45 (out of 599) 1 LAC Clean Sample sources are missing in 2LAC. Due to variability, misaligned AGNs as 3C 78, 3C 111 and 3C 120 don't make the significance cut over the 24 month period covered by the 2LAC while they were present in the 1LAC. Fornax A and Cen B are new entries relative to 1LAC.

All (except for a few) FSRQs for which $v_{s y n}$ can be estimated from archival data are of the LSP type. For BL Lacs, the breakdown is 61 LSPs, 81 ISPs, and 160 HSPs. In line with the trend already observed in the BL Lac/FSRQ ratio, HSPs constitute the most abundant subclass.

The LAT-detected FSRQs all exhibit soft $\gamma$-ray spectra while BL Lacs are more diverse as illustrated in Figure 3, showing the spectral photon index measured with the LAT plotted versus the estimated position of the synchrotron peak.

\subsection{Redshift distribution}

The redshift distributions for FSRQs and BL Lacs in the Clean Sample are presented in Figure 4 . Note that only $44 \%$ of $2 \mathrm{LAC}$ BL Lacs have measured redshifts. This is notably worse than for LBAS, in which 29 out of $42(69 \%)$ BL Lacs had measured redshifts, but similar to 1LAC $(42 \%)$. The highest redshift for a high-confidence 2LAC FSRQ is $z=3.10$. For comparison, the maximum redshift in both CGRaBS and Roma-BZCAT is for an FSRQ with $z>5$. No strong evidence for a new population of misaligned FSRQs emerging at lower redshifts is found. 


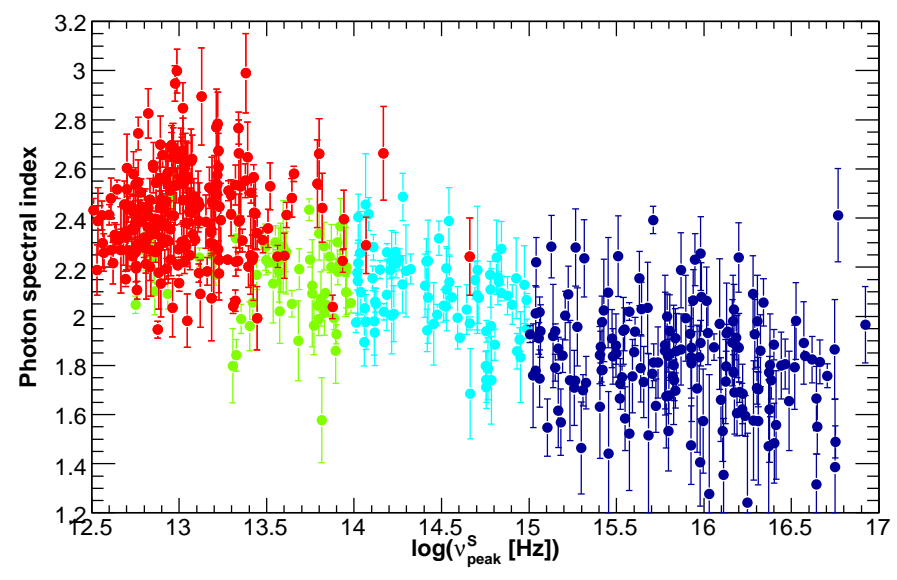

Figure 3: Peak frequency of the SED synchrotron component vs. photon spectral index for FSRQs (red) and BL Lacs (green: LSPs, cyan: ISPs, blue: HSPs) in the 2LAC Clean Sample.
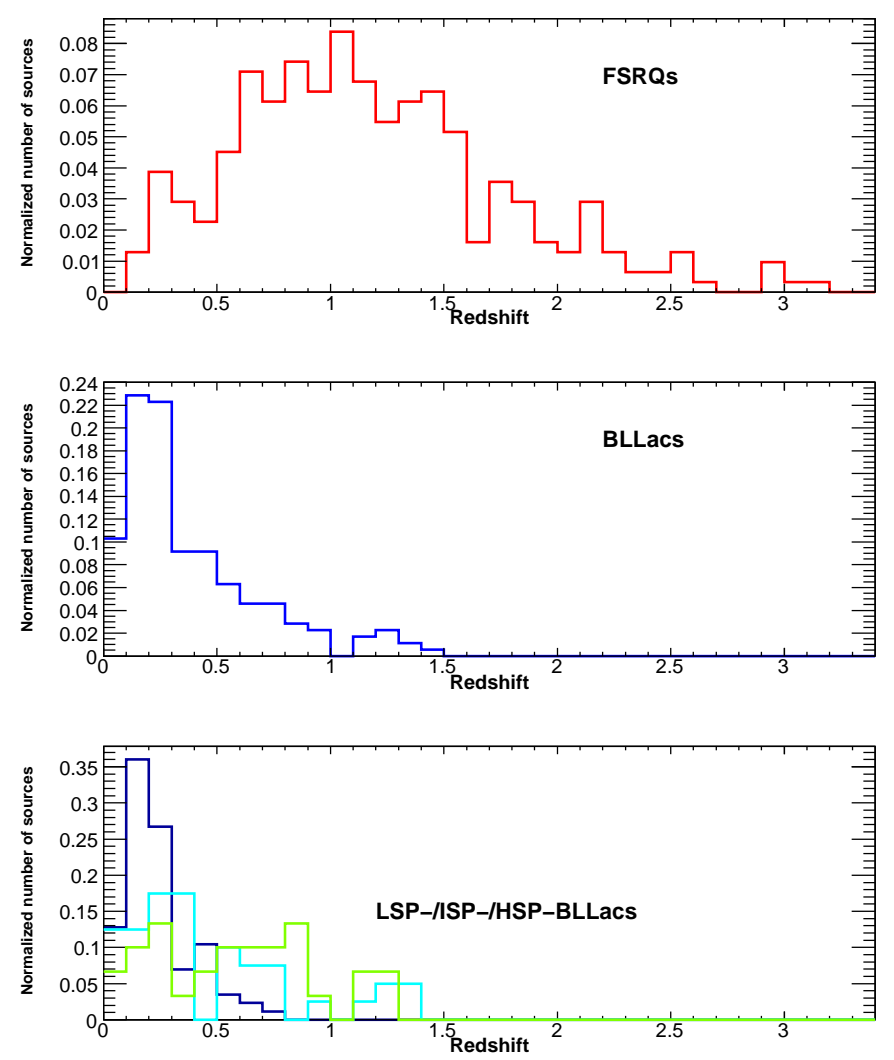

Figure 4: Top: Normalized redshift distribution for the FSRQs in the Clean Sample. Middle: The same for BL Lacs. Bottom: Redshift distributions for LSP-BL Lacs (green), ISP-BL Lacs (cyan), HSP-BL Lacs (blue) in the Clean Sample. 


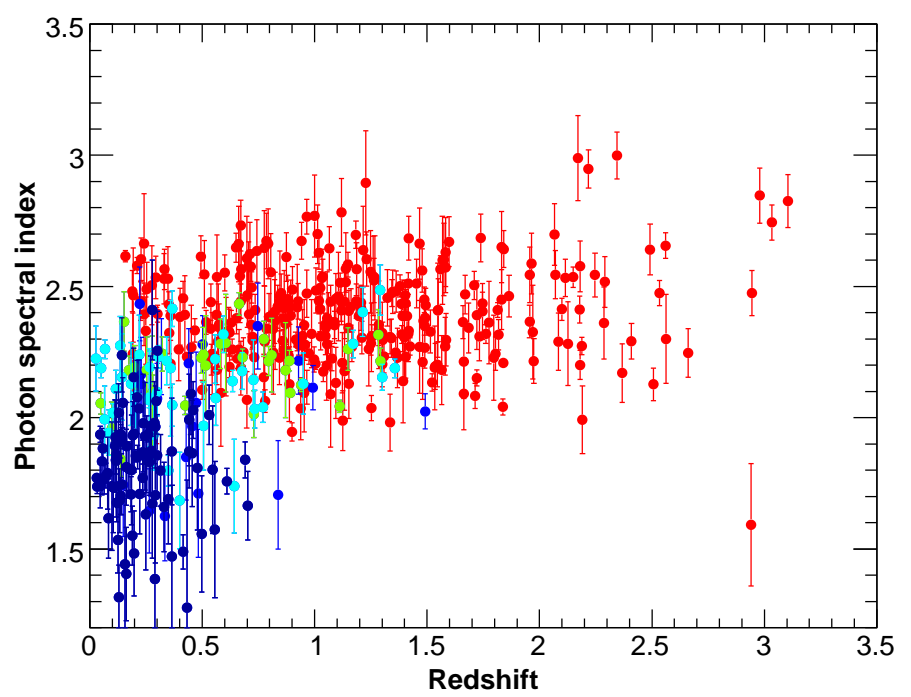

Figure 5: Redshift vs. photon spectral index vs. for FSRQs (red) and BL Lacs (green: LSPs, cyan: ISPs, blue: HSPs) in the Clean Sample.

It is interesting to compare the redshift distribution of the LAT blazars (in particular, the FSRQs) with that of the objects detected by BAT on Swift [13]. In the BAT survey, $40 \%$ of all FSRQs have $z>2$, and the distribution extends to $z \sim 4$. This behavior may be indicative of a shift in the SED peak frequencies toward lower values (i.e., a "redder" SED) for blazars at high redshifts. Indeed, the jets of the high-redshift BAT blazars are found to be more powerful than those of the LAT blazars and are among the most powerful known [14]. The peak of the inverse Compton flux for these objects, estimated to be in the $\mathrm{MeV}$ or even sub-MeV range, is located closer to the BAT band than to the LAT band, and the LAT instead samples the cutoff region of the inverse Compton spectrum.

The photon spectral index is plotted against redshift in Figure 5. For FSRQs, no significant evolution is visible. This behavior is compatible with what was previously observed for LBAS and 1LAC. The attenuation effect of the extragalactic background light (EBL) would tend to introduce spurious evidence of evolution, but the soft spectra of FSRQs and the common presence of spectral breaks at a few $\mathrm{GeV}$ [15] both minimize this effect. A stronger evolution is seen for BL Lacs: hard sources are mostly located at low redshifts, while most high-redshift sources are softer than average (though it is important to bear in mind that most BL Lacs do not have measured redshifts).

\subsection{Spectral break}

While all AGNs spectra measured with EGRET were consistent with single power law distributions, the extended energy range covered by the Fermi/LAT has enabled to discover the presence of a spectral break at a few GeV for bright LBAS FSRQs and some LSP-/ISP-BL Lacs [15]. So far, no break has ever been found in the spectrum of any bright HSP-BL Lacs, while if present it would have easily be detectable given the relative hardness of these sources. The first evidence for 


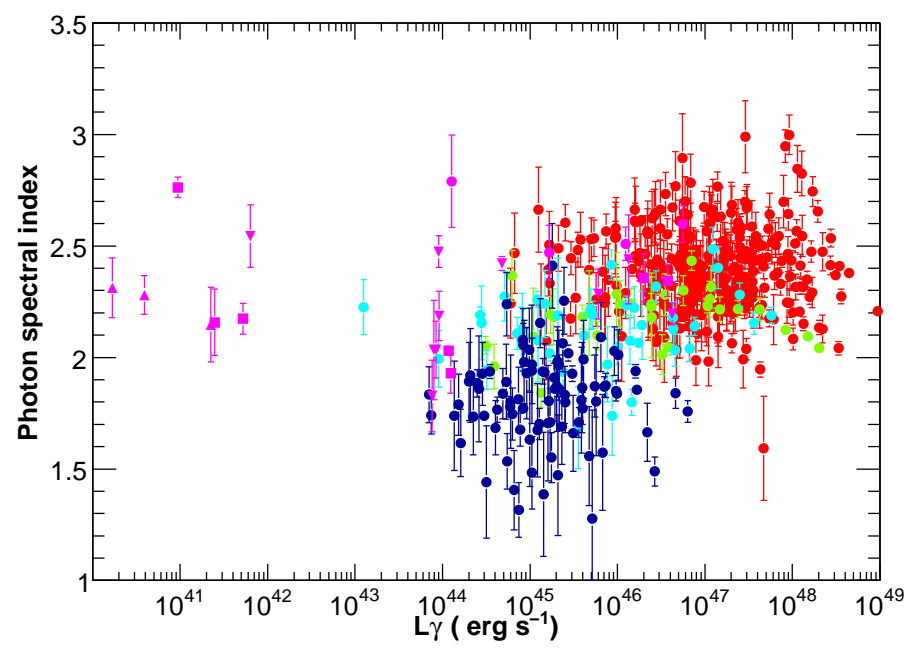

Figure 6: Photon spectral index vs. $\gamma$-ray luminosity for the different AGN classes (solid circles: FSRQs, squares: LSP-BL Lacs, triangles: ISP-BL Lacs, crosses: HSP-BL Lacs, stars: radio galaxies) in the Clean Sample.

such feature was found in $3 \mathrm{C} 454.3$ [16], which is still so far the best studied case thanks to its high recent brightness. A good model to the data is a broken power law with a break energy of $2.5 \mathrm{GeV}$ and a change in spectral slope of about 1 . The break origin is still unknown, several possibilities are being actively discussed: feature if the underlying electron energy distribution [16], superposition of two external Compton components [17], attenuation due to pair-production with He II line photons from the broad-line region [18].

In the 2LAC Clean Sample, 69 bright sources (57 FSRQs and 12 BL Lac LSPs) show very significant curvature. One must keep in mind that in order to show a high curvature index a source needs both to have a spectrum deviating from a power-law distribution and be bright enough so that the observed deviation is statistically significant. Some fainter sources thus probably do exhibit curved spectra as well.

\section{Luminosity Distributions}

Figure 6 shows the photon spectral index plotted against the $\gamma$-ray luminosity. This correlation has been widely discussed in the context of the "blazar divide" or "blazar sequence" $[19,20,21,22,23]$. It is important to bear in mind important caveats when interpreting this correlation. For instance, more than half of the BL Lacs lack measured redshifts. Strongly beamed emission can overwhelm the atomic line radiation flux and might preferentially arise from high luminosity, high redshift BL Lac objects [24]. These would then be absent in the spectral index/luminosity diagram (Fig. 6) and skew the correlation. Until the redshift incompleteness, the nature of the unassociated sources in the 2LAC, and underlying biases introduced by using different source catalogs are resolved, conclusions about the blazar sequence $[25,26]$ and the blazar divide [19] remain tentative. It is also worthwhile to mention that the correlation visible for blazars in Figure 6 is very weak if FSRQs and BL Lacs are considered separately. 


\section{Radio properties}

All 2LAC sources are radio-loud. As compared to the bulk of sources in the $8.4 \mathrm{GHz}$ CRATES catalog [8], sources with 2LAC associations cluster at the high end of the flux distribution [4], and account for nearly all the brightest CRATES sources, confirming earlier results [27, 28]. The median flux densities are 581 and 86 mJy for FSRQs and BL Lacs respectively. In their overlapping flux ranges, the distributions observed at $8 \mathrm{GHz}, 20 \mathrm{GHz}$ (ATCA [29]) and $30 \mathrm{GHz}$ (Planck [30]) are remarkably similar, evidencing the flatness of the radio spectrum. The $8 \mathrm{GHz}$ luminosity distributions are well distinct for the different blazar classes, fairly narrow and peaking around $10^{44.5}$ erg/s for FSRQs while being broader and peaking around $10^{42} \mathrm{erg} / \mathrm{s}$ for BL Lacs.

\section{Radio- $\gamma$-ray connection}

Since the start of the Fermi mission, many studies have explored the connection between the kinematic properties of the VLBA/VLBI jet and the $\gamma$-ray loudness. These studies have established that the jets of the LAT-detected blazars have:

- higher-than-average apparent speeds [31, 32],

- larger-than-average apparent opening angles [33],

- more compact radio cores [34],

- strong polarization near the base of the jet [35],

- higher variability Doppler factors [36].

All the above observations point to higher-than-average Doppler boosting in $\gamma$-ray bright blazars.

AGN jets have been found to be in a more active radio state within several months of the LAT-detection of their strong $\gamma$-ray emission $[34,37]$. The $\gamma$-ray photon flux correlates with the parsec-scale radio flux density $[34,38]$. Cross-correlating the $15.4 \mathrm{GHz}$ radio-flux density with the $\gamma$-ray fluxes provided in the 1FGL monthly light curves for 183 MOJAVE sources, Pushkarev et al. [37] found the cross-correlation function peaks at 1.2 months with the $\gamma$-rays leading. Moreover, the $\gamma$-ray loudness correlates with position of synchrotron peak and $\gamma$-ray photon spectral index for BL Lacs [39, 40].

Where does the $\gamma$-ray emission take place? Light can be shed on this important question by investigating the connection between $\gamma$-ray flares and the emergence of new superluminal radio knots. Marscher et al. [41] found that multiple $\gamma$-ray and optical flares occur in the quasar PKS 1510-089 in 2009 before the disturbance propagating down the jet passes though the mm-wave core to emerge as a new knot, as seen in the VLBA images at $43 \mathrm{GHz}$. More generally, they investigated the correlation between the $\gamma$-ray flux, the radio flux of the $43 \mathrm{GHz}$ VLBI core and the emergence of knots for $34 \gamma$-ray bright blazars and radio-galaxies [42]. They found that $2 / 3$ of the $\gamma$-ray flares were associated with superluminal knots. The opening angle of the jet [43] and the travel time associated with long duration radio events and swings in polarization angles (e.g., [41]) indicate that the $43 \mathrm{GHz}$ core and gamma-ray emitting region are greater than several $\mathrm{pc}$ from the black hole, which is in conflict with the paradigm that the $\gamma$-ray emission takes place 
within the broad-line region. The short variability time scales as well the seed photons for the external Inverse-Compton component required to properly model the SED remain a challenge to be explained within this picture.

\section{Conclusion-prospects}

The Fermi mission is still going strong, with a 4-year catalog in the works. So far the deterioration rate of the detector has been extremely slow, with fewer than 1000 (out of 880 000) tracker strips being disabled. There is no consumable aboard, so the only factor limiting the mission lifetime will probably be funding. The 2012 NASA Senior Review recommended that the mission be extended at least till $2016^{1}$. It put special emphasis on the LAT-Fermi being at the forefront of "Time-Domain Astronomy". In the long-run, the Fermi-LAT will remain extremely valuable for monitoring variable sources or detecting transient events.

\section{Acknowledgements}

The Fermi LAT Collaboration acknowledges generous ongoing support from a number of agencies and institutes that have supported both the development and the operation of the LAT as well as scientific data analysis. These include the National Aeronautics and Space Administration and the Department of Energy in the United States, the Commissariat à l'Energie Atomique and the Centre National de la Recherche Scientifique / Institut National de Physique Nucléaire et de Physique des Particules in France, the Agenzia Spaziale Italiana and the Istituto Nazionale di Fisica Nucleare in Italy, the Ministry of Education, Culture, Sports, Science and Technology (MEXT), High Energy Accelerator Research Organization (KEK) and Japan Aerospace Exploration Agency (JAXA) in Japan, and the K. A. Wallenberg Foundation, the Swedish Research Council and the Swedish National Space Board in Sweden.

\section{References}

[1] Abdo, A. A. et al. 2011, ApJL, 733, L26

[2] Hartman, R. C. et al. 1999, ApJS, 123, 79

[3] Striani, E., et al. 2010, ApJ, 718, 455

[4] Ackermann, M. et al. 2011, ApJ, 743, 171

[5] Abdo, A. A. et al. 2011, submitted to ApJS, arXiv: 1108.1435

[6] Abdo, A. A. et al. 2009, ApJ, 700, 597

[7] Abdo, A. A. et al. 2010, ApJ, 715, 429

[8] Healey, S. E. et al. 2007, , ApJS, 171, 61

[9] Healey S. E. et al. 2008, ApJS, 175, 97

[10] Massaro E. et al. 2009, A\&A, 495, 691

\footnotetext{
${ }^{1}$ http://science.nasa.gov/media/medialibrary/2012/04/03/Report_of_the_2012_Senior_Review_of_Astrophysics_Division_Operating_Missions.pdf
} 
[11] Shaw, M.S., et al., arXiv:1301.0323

[12] Abdo, A. A. et al. 2010, ApJ, 716, 30

[13] Ajello, M., et al. 2009, ApJ, 699, 603

[14] Ghisellini, G., Della Ceca, R., Volonteri, M., et al. 2010, MNRAS, 405, 387

[15] Abdo, A. A. et al. 2010, ApJ, 710, 1271

[16] Abdo, A. A. et al. 2009, ApJ, 699, 817

[17] Finke, J. D., \& Dermer, C. D. 2010, ApJL, 714, L303

[18] Poutanen, J., \& Stern, B. 2010, ApJL, 717, L118

[19] Ghisellini, G., Maraschi, L., \& Tavecchio, F. 2009, MNRAS, 396, L105

[20] Padovani, P., Giommi, P. Rau, A., 2012, MNRAS, 422, 48

[21] Meyer, E.T. et al., 2012, ApJ, 752, 4

[22] Ghisellini, G. et al., MNRAS, 425, 1371

[23] Finke, J., arXiv:1212.0869

[24] Giommi, P., Padovani, P., Polenta, G., et al. 2011, MNRAS in press (arXiv:1110.4706).

[25] Fossati, G., Maraschi, L., Celotti, A., Comastri, A., \& Ghisellini, G. 1998, MNRAS, 299, 433

[26] Ghisellini, G., Celotti, A., Fossati, G., Maraschi, L., \& Comastri, A. 1998, MNRAS, 301, 451

[27] Ghirlanda, G., Ghisellini, G., Tavecchio, F., \& Foschini, L. 2010, MNRAS, 407, 791

[28] Ackermann, M. et al. 2011, ApJ, 741, 30

[29] Murphy, T., et al. 2010, MNRAS, 402, 2403

[30] Planck Collaboration et al. 2011, A\&A, 536, A7

[31] Lister, M. L., Homan, D. C., Kadler, M., Kellermann, K. I., Kovalev, Y. Y., Ros, E., Savolainen, T., Zensus, J. A. 2009, ApJ, 696, L22

[32] Piner, B. G,. et al, 2012, ApJ, 758, 84.

[33] Pushkarev, A. B., Kovalev, Y. Y., Lister, M. L., Savolainen, T. 2009, A\&A, 507, L33

[34] Kovalev, Y. Y., et al. 2009, ApJ, 696, L17

[35] Linford, J. D., et al. 2011, ApJ, 726, 16

[36] Savolainen, T., Homan, D. C., Hovatta, T., Kadler, M., Kovalev, Y. Y.,

[37] Pushkarev, A. B., Kovalev, Y. Y., Lister, M. L. 2010, ApJ, 722, L7

[38] Arshakian, T. G., Leon-Tavares, J., Boettcher, M., Torrealba, J., Chavushyan, V. H., Lister, M. L., Ros, E., Zensus, J. A., 2012, A\&A, 537, 32

[39] Lister, M. L. et al. 2011, ApJ, 742, 27

[40] Linford, J. D., Taylor, G. B., Schinzel, F. K., 2012, ApJ, 757, 25

[41] Marscher, A.P., et al., 2010, ApJ, 710, L126

[42] Marscher, A.P., et al., arXiv:1204.6707

[43] Agudo, I., et al., 2011, ApJ, 735, L10 